34

35

\title{
Self-amplified BDNF transcription is a regulatory system for synaptic maturation in cultured
} cortical neurons

Shingo Nakajima ${ }^{1,5}$, Tadahiro Numakawa ${ }^{1,2^{*}}$, Naoki Adachi ${ }^{1,6}$, Yoshiko Ooshima ${ }^{1,3}$, Haruki Odaka ${ }^{1,4}$, Aya Yoshimura ${ }^{1}$, Hiroshi Kunugi ${ }^{1}$

1Department of Mental Disorder Research, National Institute of Neuroscience, National Center of Neurology and Psychiatry (NCNP), Tokyo, Japan

2Department of Cell Modulation, Institute of Molecular Embryology and Genetics, Kumamoto University, Kumamoto, Japan

3Department of Molecular Pharmacology, National Institute of Neuroscience, National Center of Neurology and Psychiatry (NCNP), Tokyo, Japan

4Department of Life Science and Medical Bioscience, School of Advanced Science and Engineering, Waseda University, Tokyo, Japan

5Faculty of Health Science, Hokkaido University, Sapporo, Japan

6Department of Biomedical Chemistry, Kwansei Gakuin University, Sanda, Japan

*Tadahiro Numakawa, Ph.D. (corresponding author)

Department of Mental Disorder Research, National Institute of Neuroscience, National Center

of Neurology and Psychiatry, 4-1-1, Ogawa-Higashi, Kodaira, Tokyo, 187-8502, Japan

Tel: +81-42-341-2711 ext. (5132), Fax: +81-42-346-1744,

E-mail: numakawa.yyrmk@gmail.com

\section{Running title;}

The role of self-amplifying of BDNF in neurons

\section{Keywords;}

BDNF, ERK, CREB, TrkB 


\section{Abstract}

2 Neuronal cell survival and synaptic plasticity are controlled through expression of various 3 neurotrophic factors including brain-derived neurotrophic factor (BDNF). In the present study, we 4 examined the mechanism behind BDNF-induced Bdnf mRNA production and the physiological role 5 of its amplification system using cortical neurons. Exogenous BDNF was applied to the cultured 6 cortical neurons at days in vitro (DIV) 3 and DIV 7 with or without inhibitors for intracellular 7 signaling. Expression levels of total Bdnf and Bdnf variants (exon I, exon IV, and exon $V I$ ) were 8 biphasically increased after the BDNF application in different developing stage of neurons. Inhibitor 9 for extracellular signal-regulated kinase, calmodulin dependent protein kinase II, or protein kinase A 10 repressed the BDNF-induced Bdnf mRNA expression. Furthermore, we found that application of 11 TrkB-Fc, which scavenges produced endogenous BDNF, resulted in weakened BDNF/TrkB 12 signaling and decreased expression of postsynaptic proteins, suggesting that newly synthesized 13 BDNF induced by the self-amplification system contributes to the synaptic maturation and function. 


\section{Introduction}

Brain-derived neurotrophic factor (BDNF) is a pivotal regulator for neuronal development and function such as cell survival and synaptic plasticity (Park and Poo 2013). The expression of BDNF is regulated via neuronal activity at transcriptional levels. For example, stimulation with $\mathrm{KCl}$ and kainic acid increase mRNA levels of Bdnf and reduced neuronal activity with NMDA receptor antagonists downregulates Bdnf mRNA (Zefra et al. 1990; Katanuma et al. 2014). It has been reported that, among nine promoters existing in upstream of 5'-untranslated region (UTR) of Bdnf, promoters I and IV are highly responsive to the neuronal activity due to their multiple $\mathrm{Ca}^{2+}$ - and cyclic adenosine monophosphate (cAMP) responsive elements (Pruunsild et al. 2011).

Exogenous BDNF application increases Bdnf exon IV (Yasuda et al. 2007; Zheng and Wang. 2009) in cultured cortical neurons. BDNF binding to TrkB, a high-affinity receptor for BDNF, induces the activation of downstream signals including extracellular signal-regulated kinase (ERK), phosphatidylinositol-3 kinase (PI3K)/Akt, and phospholipase C-gamma (PLC $\gamma$ ) (Park and Poo 2013; Leal et al. 2014). Activation of L-type voltage-gated calcium channel and ERK signal by pulsed electromagnetic field upregulate protein levels of BDNF in neonatal dorsal root ganglion neurons ( $\mathrm{Li}$ et al. 2014). Not only activity-dependent system, but also ERK contributes to the regulation of BDNF-induced Bdnf exon transcription in cortical neurons (Yasuda et al. 2007; Zheng and Wang 2009).

It has been suggested that activity-dependent synthesis and secretion of BDNF in both axon and dendrite is required for synaptic function including late-phase spine maturation (Kuczewski et al. 2010; Jakawich et al. 2010). Recent high-resolution visual analysis with stochastic optical reconstruction microscopy has revealed that high abundance of BDNF in glutamatergic presynapse of hippocampal neurons (Andreska et al. 2014). The activation of postsynaptic BDNF/TrkB systems also plays a role in the synaptic maturation and plasticity (Yoshii and Constantine-Paton 2010). Secreted BDNF acts as an autocrine regulator to enhance axon differentiation through the elevation of local cAMP levels and activation of protein kinase A (PKA) (Cheng et al. 2011). It has been demonstrated that the positive feedback loop of BDNF signaling contributes to hippocampal memory consolidation in rats in inhibitory avoidance test (Bambah-Mukku et al. 2014). Although these results suggest an importance of amplifying BDNF/TrkB signaling in the neuronal function, it is still unclear the contribution of accelerated self-induced BDNF production on BDNF-dependent synaptic regulation in cortical system.

Here, using cortical neurons at different developing stages, we explored a contribution of BDNF/TrkB signaling to BDNF-induced Bdnf mRNA expression and physiological role of produced BDNF in synaptic function. 
2. Materials and Methods

$2 \quad 2.1$ Cell cultures

3 Cultured cortical neurons were prepared from cerebral cortex of postnatal Wistar rats (Japan SLC,

4 Inc., Shizuoka, Japan) at the age of 1-2 days as described previously (Numakawa et al. 2009).

5 Briefly, dissected cortical tissues were digested with 9 units/ml papain and 200 units/ml DNase I for

$620 \mathrm{~min}$ at $37^{\circ} \mathrm{C}$. After the removal of enzyme solution, dissociated cortical cells with pipetting was

7 cultured with DMEM/F12 medium containing 5\% fetal bovine serum, $5 \%$ horse serum, $18 \mathrm{mg} / \mathrm{ml}$

8 streptomycin and 18 units $/ \mathrm{ml}$ penicillin. The cells were seeded on $3.5 \mathrm{~cm}$ dishes and 24 -well plates

9 at density of $5 \times 10^{5}$ cells $/ \mathrm{cm}^{2}$. After 1 day in vitro (DIV), the culture medium was replaced to

$10 \mathrm{DMEM} / \mathrm{F} 12$ medium containing 5\% horse serum with AraC (cytarabine, $2 \mu \mathrm{M}$ ). BDNF (kindly

11 gifted from Takeda Pharmaceutical company Ltd., and Sumitomo Co. Ltd.) or forskolin (Wako,

12 Japan) was applied to cortical neurons at DIV 3-4 or 6-7 with/without 20 min pretreatment of U0126

13 (Cell signaling Technology Inc., MA, USA), LY294002 (Cell signaling), CREB (cAMP responsive

14 element-binding protein) binding protein (CBP)-CREB interaction inhibitor (Calbiochem, MA,

15 USA), KN-93 (Calbiochem), H-89 (Calbiochem), and K252a (Enzo Life Science, Inc., NY, USA).

16 Recombinant Human TrkB-Fc Chimera (R\&D systems Inc. Minneapolis, MN, USA) was added at 3

17 hours after the $10 \mathrm{ng} / \mathrm{ml}$ of BDNF application (DIV 6-7) considering of TrkB-Fc action to scavenge

18 endogenous BDNF with/without washout of exogenous BDNF from the cultured medium. These

19 experiments were performed several times using different series of cultures. These experiments were

20 approved by the Ethics Review Committee for Animal Experimentation of the National Insititute of

21 Neuroscience, Japan (approval number; 2011019).

2.2 Real-time PCR

25

Total RNA was extracted using RNeasy mini kit (Qiagen, CA, USA) according to manufacturer's protocol. The reverse transcription of total RNA (100 ng) was performed with SuperScript ${ }^{\circledR}$ VIRO $^{\text {TM }}$ cDNA synthesis kit (Life Technologies Inc., CA, USA). Obtained cDNA with each specific Taqman ${ }^{\circledR}$ probe of Bdnf (Rn02531967_s1), Bdnf exon I (Rn01484924_m1), Bdnf exon IV (Rn01484927_m1), Bdnf exon VI (Rn01484928_m1), Map2 (microtubule associated protein 2, Rn00565046_m1), Ngf (nerve growth factor, Rn01533872_m1), or Gapdh (4352338E) was applied to real-time PCR system (Life Technologies Inc.). The data were analyzed using SDS 2.2 real-time PCR data analysis software (Life Technologies Inc.). The expression value of target gene was normalized by expression levels of Gapdh in each sample, then relative expression against to that of control were calculated.

\subsection{Western blotting}

36 Total cell lysates were prepared with lysis buffer containing $1 \%$ SDS, $10 \mathrm{mM}$ Tris-HCl, $10 \mathrm{mM}$ 
$1 \mathrm{Na}_{4} \mathrm{P}_{2} \mathrm{O}_{7}, 10 \mathrm{mM} \mathrm{NaF}, 5 \mathrm{mM}$ EDTA, $2 \mathrm{mM} \mathrm{Na}_{3} \mathrm{VO}_{4}$ and $1 \mathrm{mM}$ PMSF. The concentration of each

2 sample was determined by Pierce ${ }^{\circledR}$ BCA protein assay (Thermo scientific, IL, USA). The

3 equivalent amount of proteins was applied to $8 \%$ acrylamide gel, and then, separated total 4 proteins in the loaded gel were transferred to PVDF membrane (Millipore, MA, USA). The 5 primary antibody including anti-pTrk (Cell signaling, 1:500), anti-TrkB (BD biosciences, CA, 6 USA, 1:1000), anti-pERK (Cell signaling, 1:1000), anti-ERK (Cell signaling, 1:1000), 7 anti-pAkt (Cell signaling, 1:500), anti-Akt (Cell signaling, 1:1000), anti-NR2A (Sigma-Aldrich, 8 MO, USA, 1:500), anti-NR2B (Sigma-Aldrich, 1:500), anti-GluR1 (Sigma-Aldrich, 1:500), 9 anti-SNAP25 (Synaptic Systems, Germany, 1:1000), anti-synaptotagmin (BD Transduction 10 Laboratories, 1:1000), anti-syntaxin (Sigma-Aldrich, 1:3000), and anti- $\beta$-actin (Sigma-Aldrich, 11 1:5000) antibodies was applied overnight after blocking with 5\% skim milk. The secondary 12 antibody of mouse (Jackson ImmunoResearch Europe Ltd., Suffolk, UK) or rabbit (Rockland 13 Immunochemicals, Inc., Gilbertsville, PA, USA) was applied for 1 hour. After the treatment of 14 ImmunoStar® reagent (Wako Pure Chemical Industries Ltd., Japan), immunoreactivity was 15 detected by ECL films (GE healthcare UK Ltd., Buckinghamshire, UK) or AE-9150 Ez-Capture 16 II (ATTO Co., Japan). The optical density of protein expression was measured by CS Analyzer 17 version 3.00.1011 (ATTO Co.).

\subsection{Statistical analysis}

20 All data are expressed as means \pm SEM. Data were analyzed by one- or two- way ANOVA with 21 Bonferroni's multiple comparison post-hoc test (IBM Japan, Japan). Statistically significance 22 was defined as probability values less than $5 \%$. 
23.1 Biphasic increase of Bdnf mRNA expression after exogenous BDNF addition in cortical neurons

3 When BDNF (100 ng/ml, for 1, 3, 6, 12, and 24 hours) was added to both developing stage of 4 cortical neurons, endogenous levels of Bdnf mRNA was increased biphasically (see the initial peak 5 at 3 hours after BDNF addition) compared with control (no treatment; 0 hour) (Fig. 1A). We 6 confirmed dose-dependent influence of exogenous BDNF on the Bdnf mRNA expression at 3 and 24 7 hours after the BDNF application in DIV 7 (Fig. 1B). The significant effect of $10 \mathrm{ng} / \mathrm{ml}$ BDNF 8 application for 24 hours was detected by student's $t$-test $(P>0.01)$ compared to control group 9 although only higher dose of BDNF (100 and $500 \mathrm{ng} / \mathrm{ml}$ ) significantly increased Bdnf mRNA in 10 multiple comparision test. Further, mRNA levels of Bdnf variants (exon I, exon IV, and exon VI) were also increased by BDNF application similar to total Bdnf mRNA expression (Fig. 1C). Surprisingly, Bdnf exon I transcript was drastically increased after exogenous BDNF application, because Bdnf exon I transcript has been reported to be lower expressed compared to other transcripts (Katanuma et al., 2014). Activity-dependent regulation of Ngf mRNA expression has been investigated in addition to Bdnf (Zafra F et al., 1990), and MAP2 is well known as a neuronal marker (Andreska et al., 2014; Jakawich et al., 2010). To examine the specificity of exogenous BDNF application on its expression, we tested the effect of BDNF on mRNA levels of Ngf or Map2. Increase in $\mathrm{Ngf}$ or Map2 mRNA by exogenous BDNF was lower than that in Bdnf mRNA expression (Fig. 1D). Because there was no significant difference between young (DIV 3) and mature (DIV7) neurons in BDNF-induced Bdnf mRNA production, cultured cortical neurons at DIV 7 were used for subsequent experiments unless otherwise noted.

\subsection{Involvement of MAPK and PI3K/Akt signaling in the BDNF-induced Bdnf mRNA expression}

As shown in Fig. 2A, BDNF treatment significantly increased activated ERK1/2 (phosphorylated ERK1/2, pERK1/2) and Akt (phosphorylated Akt, pAkt). At 3 hours after BDNF addition, blockade of both signal cascades by MEK inhibitor (U0126) and PI3K inhibitor (LY294002) caused significant decrease in the self-induced Bdnf mRNA production by BDNF (Fig. 2B). While, at 24 hours after BDNF application, only inhibition of ERK signal with U0126 completely blocked the self-induced Bdnf mRNA production by BDNF (Fig. 2C). However, U73122, an inhibitor for PLC $\gamma$ pathway, did not show inhibitory effect on the BDNF-induced Bdnf mRNA expression at both time points (Figs. 2B and 2C).

At 3 hours after BDNF application, the expression levels of Bdnf exon I and exon IV, but not exon VI, were decreased by U0126 and LY294002 (Fig. 3A). Importantly, at 24 hours after BDNF addition, the Bdnf mRNA production from exon $I$, exon $I V$, and exon $V I$ were abolished in the presence of U0126 similar to total Bdnf mRNA expression (Fig. 3B). Although LY294002 had no 
BDNF-induced Bdnf expression from exon I and exon IV (Fig. 3B).

2

3.3 CREB and Calmodulin dependent protein kinase (CaMK) II is involved in the self-induced Bdnf mRNA production

It has been shown that activity-dependent Bdnf mRNA expression is regulated by the activation of CREB and CaMKII (Pruunsild et al. 2011). Forskolin which induces elevated cAMP levels also increased Bdnf mRNA at 3 and 24 hours after the drug application and such an increment was abolished by CBP-CREB interaction inhibitor (Fig. 4A, 4B). On the other hand, in the BDNF-induced Bdnf mRNA expression, inhibitory effect of this CBP-CREB interaction inhibitor was only observed at 24 hours after the BDNF treatment (Fig. 4A, 4B). Further, PKA inhibitor (H-89) completely abolished the self-induced Bdnf transcription at 24 hours after BDNF addition, but partial inhibitory effect by H-89 was observed at 3 hours (Fig.4A, 4B). As shown in Fig. 4C, 4D, the biphasic self-induced Bdnf mRNA production did not occur in the presence of CaMKII inhibitor (KN-93) and NMDA-type glutamate receptors inhibitor (APV).

\subsection{Blocked BDNF/TrkB signaling failed to increase of BDNF transcript}

Next, to explore the mechanism underlying late phase increase in the levels of BDNF transcript, we used TrkB inhibitors to block stimulation by endogenous BDNF produced after exogenous BDNF application. Twenty minutes pretreatment of K252a (a nonspecific Trk receptors inhibitor) significantly inhibited the BDNF-induced Bdnf mRNA expression (Fig. 5A). Although the removal of remaining exogenous BDNF in the medium at 3 hours after BDNF addition weakened the Bdnf mRNA production (12 and 24 hours, respectively) compared to no washing control, significant increase of Bdnf mRNA was still observed (Fig. 5B). Furthermore, TrkB-Fc was added at 3 hours after exogenous BDNF application to remove a contribution of BDNF including newly synthesized-endogenous BDNF. Trapping of BDNF by TrkB-Fc from 3 hours after the BDNF application blocked the increase in Bdnf mRNA levels (Fig. 5C). TrkB-Fc still had an inhibitory effect on BDNF-induced Bdnf mRNA expression after washout of exogenous BDNF (Fig. 5D). In addition, the activation of Trk and its downstream cascade including ERK- and Akt- signaling were all attenuated by TrkB-Fc treatment (Fig. 5E).

\subsection{Self-induced BDNF production plays a role in synaptic protein maintenance}

In the presence of TrkB-Fc to scavenge both exogenous BDNF and newly produced BDNF, increased postsynaptic protein glutamate receptors such as NR2A, NR2B, and GluR2/3 by BDNF application was attenuated (Fig. 6B). In contrast, no blocking effect of TrkB-Fc on expression of presynaptic proteins including SNAP25, synaptotagmin, and syntaxin was observed (Fig. 6C). 


\section{Discussion}

2 In the present study, we found biphasic self-amplification of BDNF in different developing stages

3 (DIV 3 and DIV 7) of cortical neurons. Signaling cascade via BDNF/TrkB including ERK regulated

4 the self-induced Bdnf mRNA production. In our cortical neurons, sustainable activity of BDNF/TrkB

5 signaling including self-induction contributed to its self-amplifying and upregulation of postsynaptic 6 proteins.

It has been reported that the transcriptional activity of $B d n f$ exon $I V$ is regulated by exogenous

8 BDNF treatment and activity-dependent stimuli (Yasuda et al. 2007; Zhang and Wang 2009). ERK is

9 a major signaling molecule to increase Bdnf IV transcript when exogenous BDNF is applied (Yasuda

10 et al. 2007; Zheng and Wang 2009). Although PI3K signaling partially contributed to the

11 BDNF-induced total Bdnf mRNA, our results also demonstrated that ERK is a main contributor to

12 the self-production of Bdnf mRNA. Remarkably, upregulation of Bdnf exon I when exposing to activity-dependent stimuli (including BDNF treatment) is higher compared to exon IV and exon VI, although endogenous levels of exon $I$ is much lower than exon IV and exon VI (Pruunsild et al. 2011; Katanuma e al., 2014). The inconsistency of Bdnf mRNA expression levels among experiments would reflect the difference in synaptic maturity and/or culture condition, which also supports the importance of neuronal activity for Bdnf transcription. On the other hand, U0126- or LY294002-reduced expression of exon I and exon IV, highly responsive regions to the neuronal activity, incompletely reflected to total Bdnf mRNA expression, supporting that BDNF-induced Bdnf expression is regulated via several systems but not only in activity-dependent manner.

CaMK I, II, and IV are also regulator for the Bdnf exon IV expression after treatment of exogenous BDNF and NMDA (Zheng and Wang; 2009, Zheng et al. 2011). Calcium-responsive transcription factor $(\mathrm{CaRF})$ and $\mathrm{CREB}$ are recognized as main transcriptional factor for mRNA production of BDNF (Pruunsild et al. 2011). ERK, CaMKs (I, II, and IV), and PKA also stimulate transcriptional factors to increase Bdnf exon IV transcript (Zheng et al. 2011). Although PLC $\gamma$ is involved in an increase in intracellular calcium concentration and activates CaMK II in various cell types (Kim et al. 2004), the influx of extracellular calcium also activates CaMK II (Nakanishi and Okazawa. 2006). The inhibition of NMDA receptors by APV reduced self-induction of BDNF in the present study, implying that CaMK II activation via $\mathrm{Ca}^{2+}$ influx is required for BDNF-induced Bdnf mRNA expression. Interestingly, we found possible contribution of CREB to the BDNF-induced total Bdnf mRNA in late phase (24 hours) (not in early phase; 3 hours), while CaMK, ERK, and PKA were all involved in the Bdnf mRNA production in early phase. The regulation of BDNF levels in each time course has been demonstrated (Yasuda et al. 2007; Zheng and Wang 2009). Yasuda et al. reported that the transcript of Bdnf exon $I V$ is increased activity-dependently only in early phase

35 (Yasuda et al. 2007). Notably, exon IV transcription by NMDA or BDNF are regulated differently 36 (Zheng and Wang 2009), and we previously observed that reduced neuronal activity with blockade 
1 of NMDA receptors rapidly decreased total and exon IV mRNA of BDNF through the inactivation of 2 CREB (Katanuma et al. 2014). It is possible that BDNF/TrkB system and neural activity 3 concurrently induce various intracellular signaling in early or late phase after BDNF binding to its 4 receptor, resulting in achievement of fine control in amplifying the self-induction of BDNF.

Signaling kinetics of BDNF/TrkB system is critical aspect in regulation of neuronal function.

6 Sustained TrkB activity by gradual application of BDNF or depolarization before the BDNF 7 stimulation enhances neurite branching and spine neck elongation (Ji et al. 2010; Guo et al. 2014). In 8 the present study, the scavenging of BDNF using TrkB-Fc from the first peak of BDNF transcription 9 levels (3 hours) suggested the possible contribution of self-amplifying BDNF on the sustainable $10 \mathrm{BDNF} /$ TrkB signaling. Interestingly, self-amplifying BDNF acts as an autocrine factor to accelerate 11 axon development through local elevation of cAMP and PKA activity (Cheng et al. 2011). We found 12 that such a sustained BDNF/TrkB signaling is required for the further amplification of BDNF 13 production observed in late phase followed by upregulation of postsynaptic proteins. Because 14 postsynaptic BDNF/TrkB signaling is required for synaptic maturation and function (Yoshii and 15 Constantine-Paton, 2010), the acceleration of BDNF self-production may be important to regulate 16 synaptic plasticity. It is required that experiments such as specific downregulation of BDNF by 17 RNAi and/or stimulation with labeled BDNF to distinguish from endogenous BDNF in the future. 18 The present results have firstly demonstrated a physiological contribution of the elevated endogenous BDNF levels via self-production system which prolongs BDNF/TrkB signaling and increases postsynaptic proteins.

Decreased levels of Bdnf and Trkb mRNA expression in prefrontal cortex and hippocampus were observed in Alzheimer's disease, schizophrenia, and mood disorders (Ray et al. 2014). The positive effect of exercise on hippocampal Bdnf mRNA expression contributes to improve object recognition and to increase cell proliferation (Garza et al. 2004; Bechara and Kelly 2013). Exercise and magnetic stimulation also increases hippocampal TrkB transcript (Vaynman et al. 2003; Ma et al. 2013). Recently, it has been demonstrated that positive BDNF-loop is important for the memory consolidation mediated by the hippocampus (Bambah-Mukku et al. 2014). These studies including our present results suggest that exploring external stimuli to trigger the self-amplifying BDNF system has a beneficial effect to treat brain diseases.

\section{Acknowledgements}

32 This study was supported by grants from Takeda Science Foundation (T.N.), the Grant-in-Aid for 33 Challenging Exploratory Research (JSPS KAKENHI Grant Number 25640019) (T. N.), and the 34 Grant-in-Aid for Scientific Research (B) (JSPS KAKENHI 24300139) (T. N.) in the Ministry of 35 Education, Culture, Sports, Science, and Technology of Japan. This study also supported by the 36 Health and Labor Sciences Research Grants (H. K.), Intramural Research Grant for Neurological and 
1 Psychiatric Disorders of NCNP (H.K.) and CREST, Japan Science and Technology Agency (JST)

2 (T.N., N.A. and H.K.).

3 


\section{$1 \quad$ References}

2

5

Andreska T, Aufmkolk S, Sauer M, Blum R. (2014) High abundance of BDNF within glutamatergic presynapses of cultured hippocampal neurons. Front Cell Neurosci. 8, 107.

Bambah-Mukku D, Travaglia A, Chen DY, Pollonini G, Alberini CM. (2014) A positive autoregulatory BDNF feedback loop via C/EBP $\beta$ mediates hippocampal memory consolidation. J Neurosci. 34, 12547-12559.

Bechara RG, Kelly ÁM. (2013) Exercise improves object recognition memory and induces BDNF expression and cell proliferation in cognitively enriched rats. Behav Brain Res. 245, 96-100.

Cheng PL, Song AH, Wong YH, Wang S, Zhang X, Poo MM. (2011) Self-amplifying autocrine actions of BDNF in axon development. Proc Natl Acad Sci U S A. 108, 18430-18435.

Garza AA, Ha TG, Garcia C, Chen MJ, Russo-Neustadt AA. (2004) Exercise, antidepressant treatment, and BDNF mRNA expression in the aging brain. Pharmacol Biochem Behav. 77, 209-220.

Guo W, Ji Y, Wang S, Sun Y, Lu B. (2014) Neuronal activity alters BDNF-TrkB signaling kinetics and downstream functions. J Cell Sci. 127, 2249-2260.

Jakawich SK, Nasser HB, Strong MJ, McCartney AJ, Perez AS, Rakesh N, Carruthers CJ, Sutton MA. (2010) Local presynaptic activity gates homeostatic changes in presynaptic function driven by dendritic BDNF synthesis. Neuron. 68, 1143-1158.

Ji Y, Lu Y, Yang F, Shen W, Tang TT, Feng L, Duan S, Lu B. (2010) Acute and gradual increases in BDNF concentration elicit distinct signaling and functions in neurons. Nat Neurosci. 13, 302-309.

Katanuma Y, Numakawa T, Adachi N, Yamamoto N, Ooshima Y, Haruki O, Takafumi I, Kunugi H. (2014) Phencyclidine rapidly decreases neuronal mRNA of brain-derived neurotrophic factor. Synapse. 68, 257-265.

Kim SY, Ahn BH, Kim J, Bae YS, Kwak JY, Min G, Kwon TK, Chang JS, Lee YH, Yoon SH, Min DS. (2004) Phospholipase C, protein kinase C, Ca2+/calmodulin-dependent protein kinase II, and redox state are involved in epigallocatechin gallate-induced phospholipase $\mathrm{D}$ activation in human 
astroglioma cells. Eur J Biochem. 271, 3470-3480.

Kuczewski N, Porcher C, Gaiarsa JL. (2010) Activity-dependent dendritic secretion of brain-derived neurotrophic factor modulates synaptic plasticity. Eur J Neurosci. 32, 1239-1244.

Leal G, Comprido D, Duarte CB. (2014) BDNF-induced local protein synthesis and synaptic plasticity. Neuropharmacology. 76, 639-656.

Li Y, Yan X, Liu J, Li L, Hu X, Sun H, Tian J. (2014) Pulsed electromagnetic field enhances brain-derived neurotrophic factor expression through L-type voltage-gated calcium channel- and Erk-dependent signaling pathways in neonatal rat dorsal root ganglion neurons. Neurochem Int. 75, 96-104.

Ma J, Zhang Z, Su Y, Kang L, Geng D, Wang Y, Luan F, Wang M, Cui H. (2013) Magnetic stimulation modulates structural synaptic plasticity and regulates BDNF-TrkB signal pathway in cultured hippocampal neurons. Neurochem Int. 62, 84-91.

Nakanishi S, Okazawa M. (2006) Membrane potential-regulated Ca2+ signalling in development and maturation of mammalian cerebellar granule cells. J Physiol. 575, 389-395.

Numakawa T, Kumamaru E, Adachi N, Yagasaki Y, Izumi A, Kunugi H. (2009) Glucocorticoid receptor interaction with TrkB promotes BDNF-triggered PLC-gamma signaling for glutamate release via a glutamate transporter. Proc Natl Acad Sci U S A. 106, 647-652.

Park H, Poo MM. (2013) Neurotrophin regulation of neural circuit development and function. Nat Rev Neurosci. 14, 7-23.

Pruunsild P, Sepp M, Orav E, Koppel I, Timmusk T. (2011) Identification of cis-elements and transcription factors regulating neuronal activity-dependent transcription of human BDNF gene. $\mathrm{J}$ Neurosci. 31, 3295-3308.

Ray MT, Shannon Weickert C, Webster MJ. (2014) Decreased BDNF and TrkB mRNA expression in multiple cortical areas of patients with schizophrenia and mood disorders. Transl Psychiatry. 4, e389.

Vaynman S, Ying Z, Gomez-Pinilla F. (2003) Interplay between brain-derived neurotrophic factor 
1 and signal transduction modulators in the regulation of the effects of exercise on 2 synaptic-plasticity. Neuroscience. 122, 647-657.

3

4 Yasuda M, Fukuchi M, Tabuchi A, Kawahara M, Tsuneki H, Azuma Y, Chiba Y, Tsuda M. (2007)

5 Robust stimulation of TrkB induces delayed increases in BDNF and Arc mRNA expressions in 6 cultured rat cortical neurons via distinct mechanisms. J Neurochem. 103, 626-636.

7

8
Yoshii A, Constantine-Paton M. (2010) Postsynaptic BDNF-TrkB signaling in synapse maturation, plasticity, and disease. Dev Neurobiol. 70, 304-322.

Zafra F, Hengerer B, Leibrock J, Thoenen H, Lindholm D. (1990) Activity dependent regulation of BDNF and NGF mRNAs in the rat hippocampus is mediated by non-NMDA glutamate receptors. EMBO J. 9, 3545-3550.

Zheng F, Wang H. (2009) NMDA-mediated and self-induced bdnf exon IV transcriptions are differentially regulated in cultured cortical neurons. Neurochem Int. 54, 385-392.

Zheng F, Zhou X, Luo Y, Xiao H, Wayman G, Wang H. (2011) Regulation of brain-derived neurotrophic factor exon IV transcription through calcium responsive elements in cortical neurons. PLoS One. 6, e28441. 
3 Figure 1 BDNF-induced Bdnf mRNA expression in cultured cortical neurons.

4 (A) The expression levels of total Bdnf mRNA induced by treatment with BDNF (100 ng/mL) at

DIV 3 and DIV 7 (n=4). (B) The dose-dependent effect of BDNF treatment for 3 or 24 hours on cortical Bdnf mRNA expression at DIV 7(n=4). (C) BDNF (100 ng/ml) increased Bdnf exon I, exon

$I V$, and exon $V I(\mathrm{n}=4)$. (D) $N g$ f or Map2 mRNA expression after BDNF (100 ng/mL) application ( $\mathrm{n}=4)$. The values are mean \pm SEM. ${ }^{*} P<0.05,{ }^{* *} P<0.01,{ }^{* * *} P<0.001$ vs control. ${ }^{\#} P<0.05$.

Figure 2 Involvement of ERK and Akt signaling in the Bdnf mRNA production induced by exogenous BDNF application

(A) Time course (1, 3, 6, 12, and 24 hours) of phosphorylated ERK (pERK) and Akt (pAkt) after the treatment of $100 \mathrm{ng} / \mathrm{ml} \mathrm{BDNF}$ at DIV $7(\mathrm{n}=6)$. After stripping the antibody for pERK or pAkt, we performed re-blotting with antibody for ERK or Akt. The effect of U0126 $(10 \mu \mathrm{M}), \mathrm{U} 73122(10 \mu \mathrm{M})$, and LY294002 $(10 \mu \mathrm{M})$ on BDNF-induced Bdnf mRNA expression at (B) 3 and (C) 24 hours after BDNF addition ( $\mathrm{n}=5-6)$. The values are mean \pm SEM. ${ }^{*} \mathrm{P}<0.05,{ }^{* *} \mathrm{P}<0.01,{ }^{* * *} \mathrm{P}<0.001$ vs control, ${ }^{\dagger \dagger} \mathrm{P}<0.001$ vs solo inhibitor application, ${ }^{\# \#} \mathrm{P}<0.01,{ }^{\# \#} \mathrm{P}<0.001$.

Figure 3 Changed levels of Bdnf exon mRNA by BDNF in the presence of inhibitor for ERK or PI3K signaling.

Production of each Bdnf exon (exon I, exon IV, and exon VI) in the absence or presence of U0126 or LY294002 at 3 (A) and 24 hours (B) after BDNF addition ( $\mathrm{n}=4-6)$. The values are mean \pm SEM. ${ }^{* *} P$ $<0.01,{ }^{* * *} \mathrm{P}<0.001$ vs control, ${ }^{\dagger} \mathrm{P}<0.05,{ }^{\dagger \dagger} \mathrm{P}<0.01,{ }^{\dagger \dagger} \mathrm{P}<0.001$ vs solo inhibitor application, ${ }^{\#} \mathrm{P}<0.01,{ }^{\# \#} \mathrm{P}<0.001$.

\section{Figure 5 Self-amplifying BDNF sustained BDNF/TrkB signaling}

(A) The effect of K252a (an inhibitor for TrkB, $200 \mathrm{nM}$ ) on Bdnf mRNA expression induced by $100 \mathrm{ng} / \mathrm{ml}$ of BDNF ( $\mathrm{n}=6)$. (B) Bdnf mRNA levels still increased after exogenous BDNF (100 $\mathrm{ng} / \mathrm{ml})$ washout $(\mathrm{n}=4-5)$ at DIV 3. (C) TrkB-Fc $(1 \mu \mathrm{g} / \mathrm{ml})$ abolished the induction of Bdnf 
1 exogenous BDNF (100 ng/ml, 3 hours) from the medium also blocked the increase of Bdnf 2 mRNA levels at DIV $3(n=6)$. (E) BDNF-dependent activation of TrkB and downstream 3 signaling in the presence of TrkB-Fc. TrkB-Fc $(1 \mu \mathrm{g} / \mathrm{ml})$ was applied at 3 hours after exogenous 4 BDNF application $(10 \mathrm{ng} / \mathrm{ml})(\mathrm{n}=5-6)$. The values are mean \pm SEM. ${ }^{* *} \mathrm{P}<0.01,{ }^{* * *} \mathrm{P}<0.001 \mathrm{vs}$ 5 control, ${ }^{\#} \mathrm{P}<0.05,{ }^{\# \#} \mathrm{P}<0.01,{ }^{\# \#} \mathrm{P}<0.001$ vs in the presence of TrkB-Fc. We stripped the antibody 6 for pERK or pAkt, then, re-blotted with antibody for ERK or Akt.

7

8 Figure 6 Self-amplifying BDNF contributes to the upregulation of postsynaptic proteins

9 (A) Representative images of expression of synaptic proteins after BDNF application. (B) 10 Postsynaptic glutamate receptors (NR2A, NR2B, and GluR2/3) and (C) presynaptic proteins 11 (SNAP25, synaptotagmin, and syntaxin) after BDNF (10 ng/ml, 24 hours) treatment with or without 12 TrkB-Fc $(1 \mu \mathrm{g} / \mathrm{ml})(\mathrm{n}=8-9)$. The values are mean \pm SEM. ${ }^{*} P<0.05,{ }^{* *} P<0.01,{ }^{* * *} P<0.001$ vs 13 control, ${ }^{\#} P<0.05,{ }^{\#} P<0.01,{ }^{\# \#} \mathrm{P}<0.001$. 
A
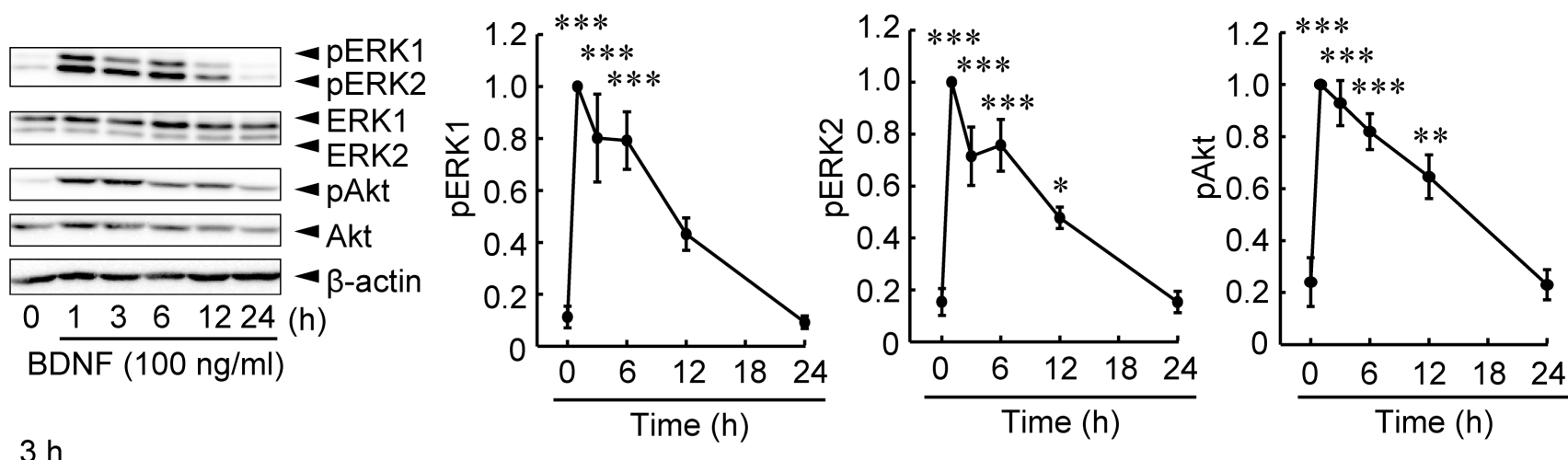

B $3 \mathrm{~h}$

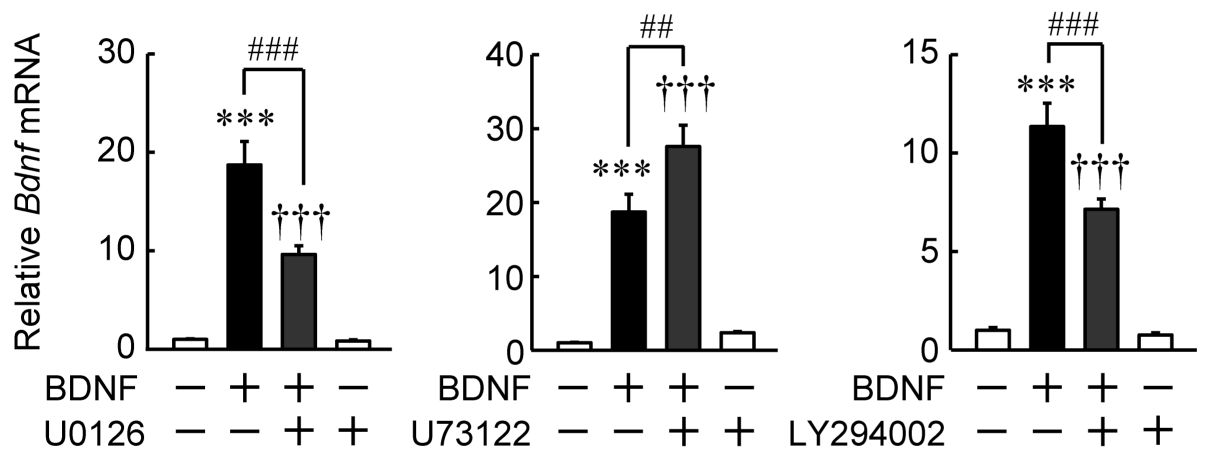

C $24 \mathrm{~h}$
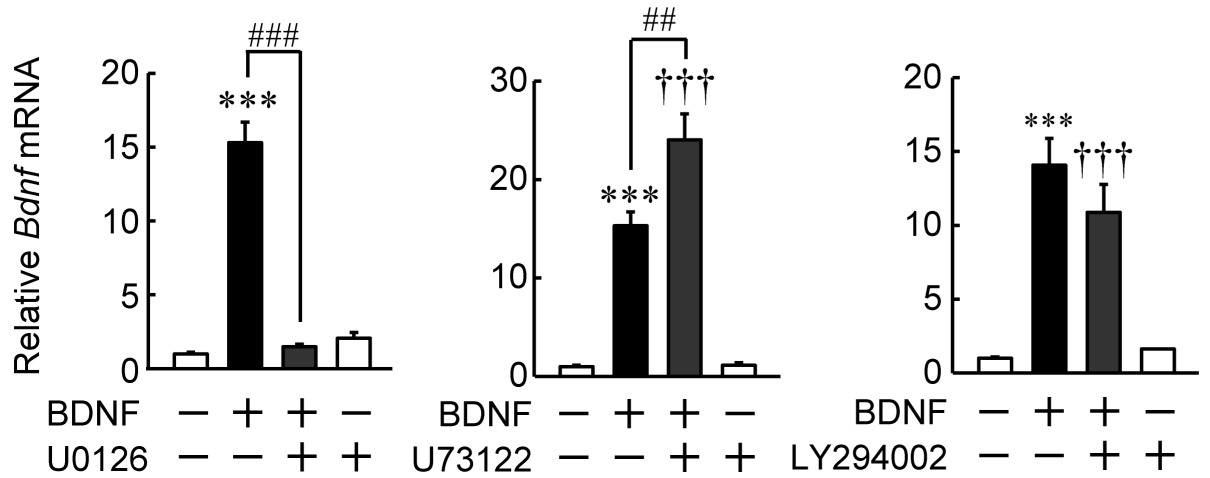

$\mathrm{U} 0126--++\mathrm{U} 73122--++\mathrm{LY} 294002--++$ 
A $3 \mathrm{~h}$

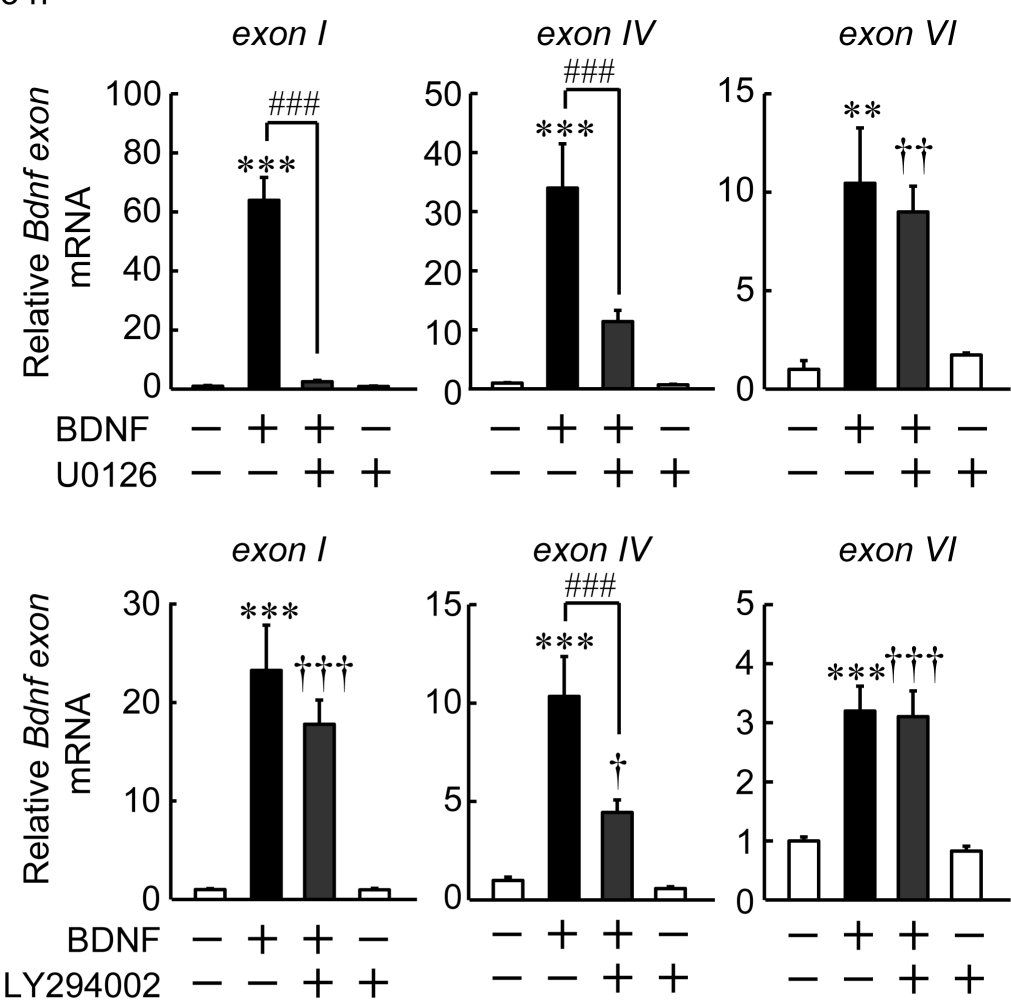

B $24 \mathrm{~h}$
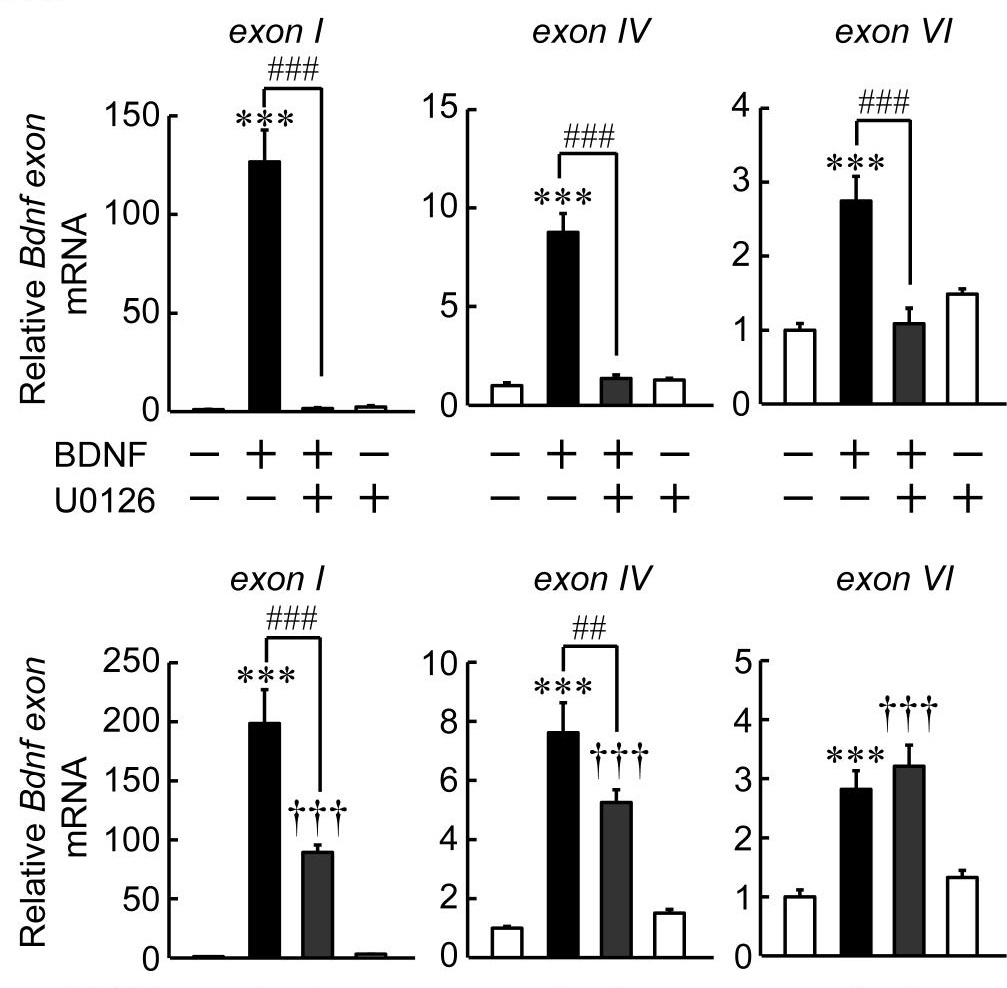

$-++-$

$--++$

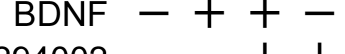

$-++-$

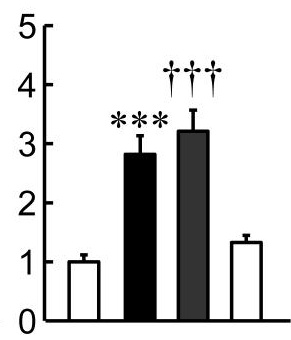

LY294002 - - + +

$-++-$

$--++$

exon VI 
A $3 \mathrm{~h}$

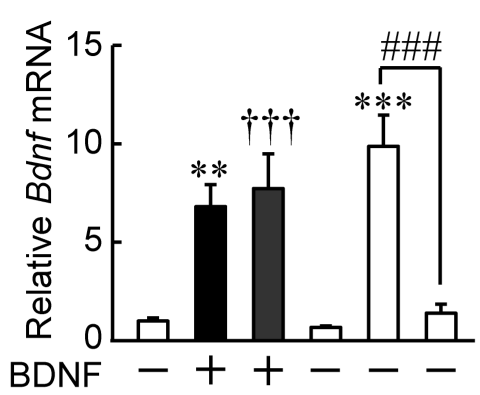
CBP-CREB --++-+
B $24 \mathrm{~h}$
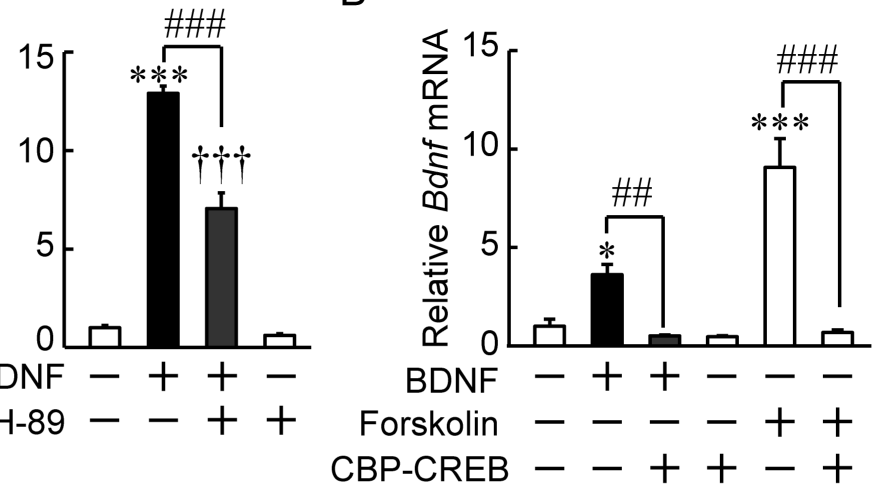

BDNF

\begin{abstract}
Forskolin
\end{abstract}
CBP-CREB --++-+
C $3 \mathrm{~h}$

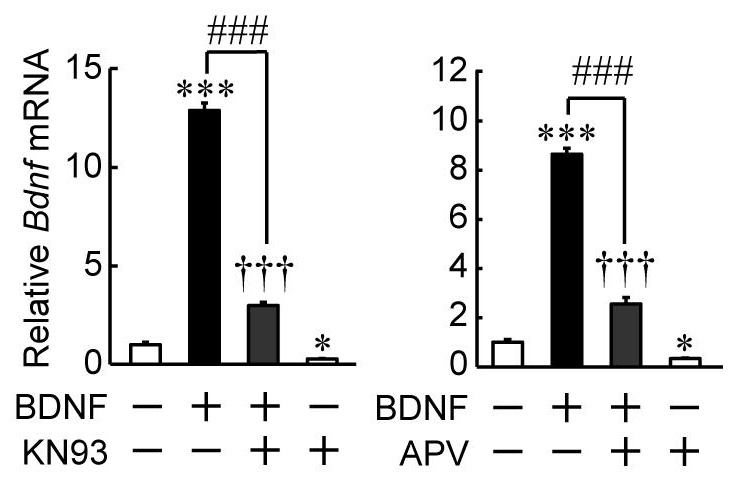

D $24 \mathrm{~h}$

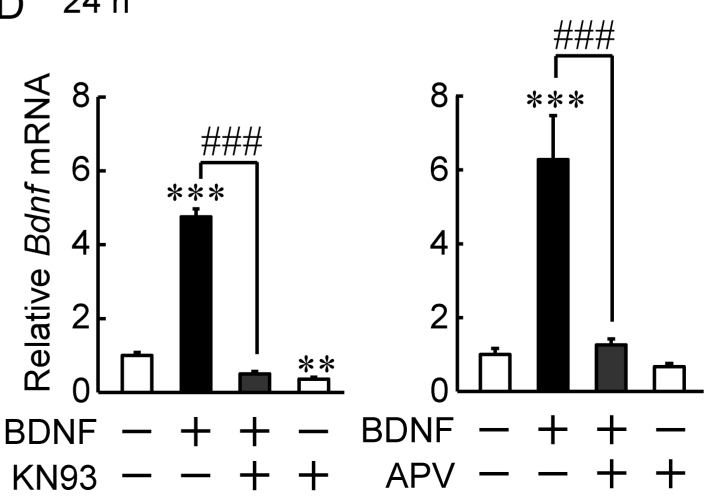




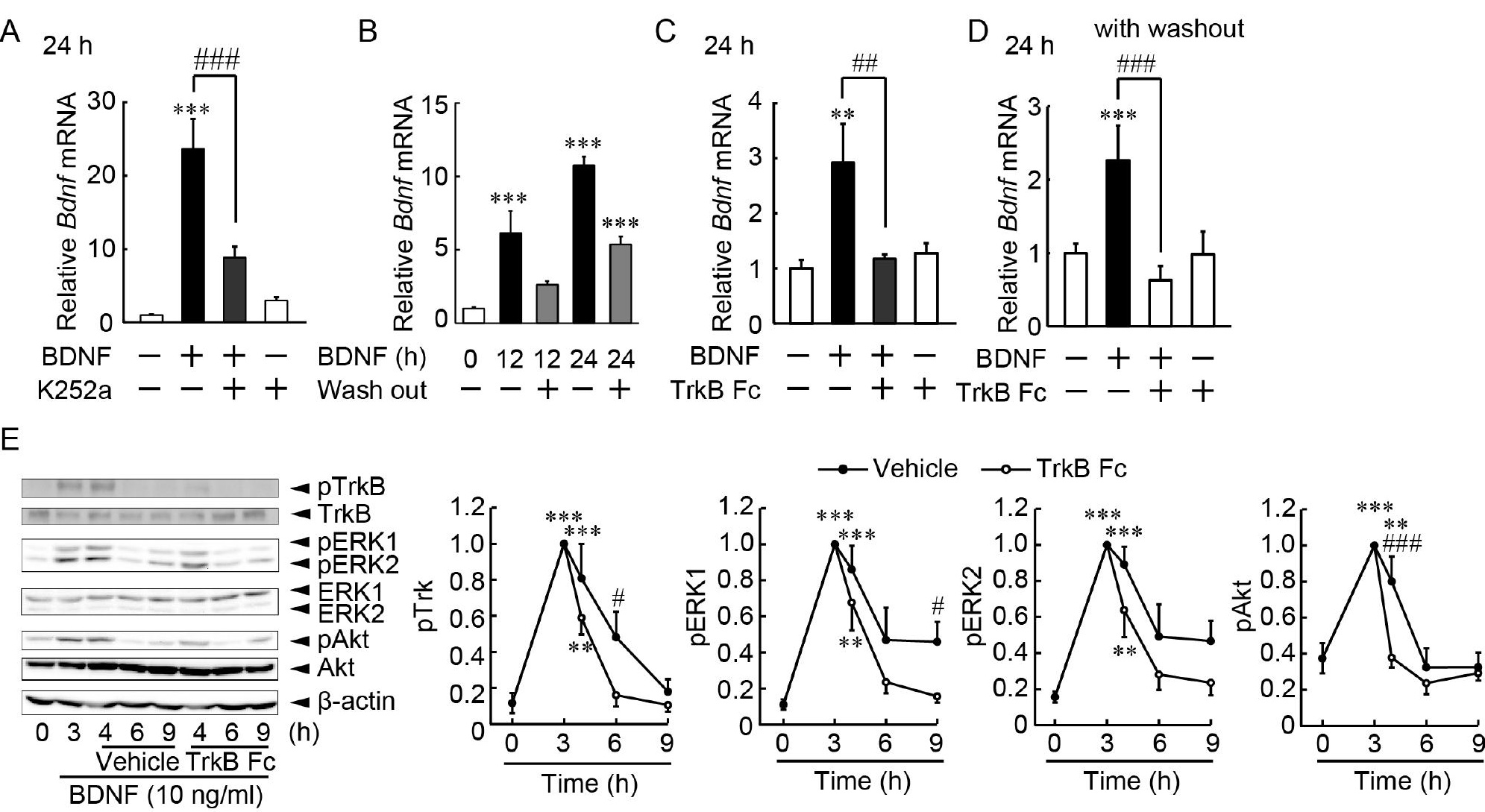



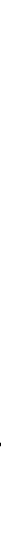

C

SNAP25

Synaptotagmin

Syntaxin
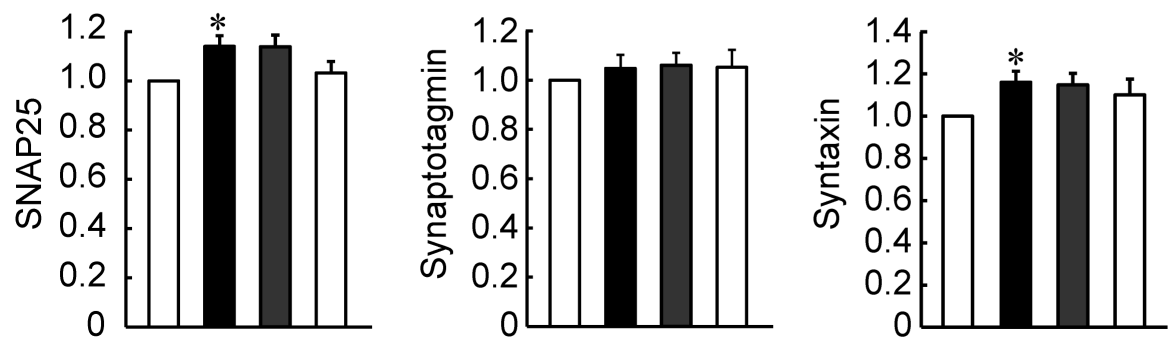

BDNF -++-

$$
\text { BDNF }-++- \text { BDNF }-++-
$$

TrkB Fc --++ TrkB Fc --++ TrkB Fc --+ 